\title{
Risk factors for social withdrawal in Amyotrophic Lateral Sclerosis /Motor Neurone Disease
}

Daniela K Schlüter ${ }^{1 *}$, Alan Tennant ${ }^{2}$, Roger Mills ${ }^{3}$, Peter J. Diggle ${ }^{1}$ Carolyn A Young ${ }^{3,4}$

On behalf of the TONiC study group

${ }^{1}$ Centre for Health informatics, Computing and Statistics (CHICAS), Lancaster Medical

School, Lancaster University, Lancaster LA1 4YW, UK

${ }^{2}$ Swiss Paraplegic Research, Nottwil, Switzerland

${ }^{3}$ Walton Centre NHS Foundation Trust, Liverpool, UK;

${ }^{4}$ University of Liverpool, $U K$

*Corresponding author; e-mail address:d.schlueter@lancaster.ac.uk

TONiC study group:

Al-Chalabi $A^{5}$, Burke $G^{6}$, Dick $D J^{7}$, Ealing $J^{8}$, Hanemann $C O^{9}$, Harrower $T^{10}$, McDermott $C J^{11}$, Majeed $T^{12}$, Pinto $A^{13}$, Talbot $K^{14}$, Williams $T^{15}$

${ }^{5}$ King's College London, UK

${ }^{6}$ Wessex Neurological Centre, UK

${ }^{7}$ Norfolk \& Norwich University Hospital, UK

${ }^{8}$ Salford Royal Foundation Trust, Manchester, UK

${ }^{9}$ Plymouth University Peninsula Schools of Medicine and Dentistry, UK

${ }^{10}$ Royal Devon and Exeter Foundation Trust Hospital, UK

${ }^{11}$ Sheffield Institute for Translational Neuroscience, University of Sheffield, UK

${ }^{12}$ Lancashire Teaching Hospitals, Preston, UK

${ }^{13}$ Wessex Neurological Centre, UK

${ }^{14}$ University of Oxford, UK

${ }^{15}$ Royal Victoria Infirmary, Newcastle upon Tyne, UK 


\section{Biographical notes}

Daniela K. Schlüter is a Senior Research Associate in Biostatistics in the Lancaster Medical School. Her research interests are in statistical methods for longitudinal data and causal inference and their applications in population health.

Alan Tennant was appointed to the Chair of Rehabilitation Studies at Leeds in 2000, he retired with Emeritus status in June 2014. Subsequently he took up an appointment as Senior Advisor with Swiss Paraplegic Research, Switzerland, together with a permanent visiting professorship at the University of Luzern.

Dr Roger Mills has been a consultant neurologist since 2011 and is co-investigator for the TONiC study.

Peter J. Diggle is Distinguished Professor in the Lancaster Medical School. His research interests are in the development of statistical methods for spatial and longitudinal data and their applications in the biomedical and population health sciences.

Professor Carolyn Young is a consultant neurologist and has lead the MND Care Centre in Liverpool since 1992. She is the UK Chief Investigator for the TONiC study. 


\begin{abstract}
Background

Greater social withdrawal is related to higher levels of psychological distress and poorer adaptation to a diagnosis of amyotrophic lateral sclerosis.
\end{abstract}

\title{
Objectives
}

To examine whether demographics and functional deficits can be used to assess which patients may be at risk of social withdrawal and whether symptoms including depression and anxiety can provide additional information for identifying individuals at risk. Furthermore, to examine whether patient-perceived stigma has a role in mediating the effects of any of the predictors of social withdrawal.

\section{Methods}

559 participants in the ongoing Trajectories of Outcomes in Neurological Conditions (TONiC) study completed a questionnaire pack collecting data on demographics and a range of patient reported measures. Multiple regression analysis was employed to assess associations of functional ability, demographics, physical symptoms, anxiety, and depression with social withdrawal. The mediating role of stigma was assessed through the development of a bivariate linear regression model for stigma and social withdrawal.

\section{Results}

Disability in the bulbar and motor domains, anxiety and depression were found to be significant predictors of social withdrawal. Stigma was the strongest single predictor of social withdrawal and was found to partially mediate the effects of functional deficits and mood on social withdrawal. 


\section{Conclusions}

Social withdrawal is associated with worse motor disability and poorer bulbar function, as well as increased anxiety and depression. Stigma is a powerful predictor for social withdrawal; further work should investigate whether stigma is a potential target for psychological interventions aimed at reducing social withdrawal and improving quality of life.

Epidemiology, Clinical trials, Prognostic 


\section{Introduction}

Amyotrophic lateral sclerosis (ALS), or motor neurone disease (MND), is a disease of progressive degeneration of the motor neurones in the brain and spinal cord causing weakness and wasting of the muscles (1). Symptoms range from difficulty walking and muscle cramps to the inability to speak and swallow, and can include paralysis and respiratory failure (2). Survival can be between a few months to, exceptionally, decades; in most cases, however, patients die within two to five years of diagnosis (1). Currently there is no cure available; therefore treatment is focussed on managing symptoms, palliative care and optimising quality of life (2).

A growing body of evidence shows that quality of life in people affected by ALS is significantly determined by psychosocial factors such as social support, ability to cope, fatigue and depression (3-5). There is a positive relationship between social support and quality of life in ALS $(4,6)$. In other neurological conditions such as Parkinson's disease and multiple sclerosis, reduced social contact is one of the factors associated with poor quality of life $(7,8)$. Social withdrawal is a complex social phenomenon often seen to arise from internal factors, with the person affected actively choosing to reduce social interaction, potentially due to increased anxiety in social situations or embarrassment $(9,10)$. Social withdrawal in ALS is associated with depression (11) and may be a mediator for the effects of fatigue and coping ability on quality of life (3). People with ALS frequently report being socially withdrawn from friends and family. Some describe contact with their professional caregivers as their most common form of social interaction (10). Understanding risk factors and potential 
causes for social withdrawal may therefore be a crucial first step to developing psychological interventions that lead to improved quality of life.

In the current study we aim to investigate more closely the association of functional status and demographic characteristics with social withdrawal in ALS to identify which factors, practicable for routine assessment by clinical staff, might highlight patients at risk of social withdrawal. In addition, we investigate whether common physical symptoms (drooling, choking, head drop, muscle cramps, muscle twitching and emotional lability), anxiety and depression, can provide additional information regarding who is at risk of social withdrawal. As embarrassment has been identified as one of the key drivers for social withdrawal in ALS (10) we investigate the hypothesis that the effects on social withdrawal are mediated by patient perceived stigma (see Supplementary Material section S.3 for the hypothesised causal pathway). Stigma is defined as an attribute or characteristic that is devalued in a particular social context (12). It is a multi-dimensional concept including the domains of enacted and internalised stigma, which are measured by the Stigma Scale for Chronic Illness (13) that we use in this study. Enacted stigma refers to the experience of negative attitudes and unfair treatments by others. If an individual perceives enacted stigma, it can be internalised leading to the individual believing that the negative public attitudes or stereotypes apply to them. Both enacted and internalised stigma can result in various negative outcomes including marginalisation, self-isolation and reduced quality of life $(13,14)$. 


\section{Material and Methods}

\section{Data collection}

Participants were recruited into the ongoing Trajectories of Outcomes in Neurological Conditions (TONiC) study from ALS clinics across the United Kingdom. Following informed consent, participants completed a questionnaire pack, for which a caregiver could act as scribe if the patient was unable to hold a pen. Demographic and diseasespecific data, as well as a range of patient reported measures, were collected in the pack. Ethical approval was granted from the relevant local research committees (reference 11/NW/0743).

\section{Rasch analysis}

All scores from ordinal questionnaires were transformed to interval-level scaling through fit of their data to the Rasch model (15). Full details of the approach are described elsewhere (16). Briefly, data from the scale should show consistent stochastic ordering of items and subjects; items should show local independence (conditioned on the raw score) (17); items should be invariant across relevant groups such as age and gender (Differential Item Functioning) and the scale (or domains) should be unidimensional (18).

Tables of fit of the Rasch model to the data for all the scales used in the analysis are given in the Supplementary Material (section S.1). 


\section{Questionnaire Measures}

\section{Social Withdrawal}

Social Withdrawal was assessed using the 14-item summary scale from the MNDSocial Withdrawal Scale (19) which ranges from 0 to 42 with lower values indicating less social withdrawal. The nomogram for conversion of raw scores into interval-level scores is shown in the Supplementary Material (section S.2).

\section{Stigma}

Stigma was assessed using the 8-item Stigma Scale for Chronic Illness (SSCI-8) (13). The scale ranges from 0-32. Lower scores indicate less stigma. The nomogram for conversion of raw scores into interval-level scores is given in the Supplementary Material (section S.2)

\section{Functional ability}

Functional ability was measured by the 12-item ALS Functional Rating Scale-Revised (ALSFRS-R) (20). The ALSFRS-R has been criticised as multidimensional, with a recommendation that it is reported as a profile of scores for Bulbar, Motor Function and Respiratory domains, rather than a total score (21). The sub-scales range from 0-12, 024 and 0-12 for the bulbar, motor and respiratory domains, respectively. Lower scores indicate greater disability.

\section{Depression and Anxiety}

The modified 12-item Hospital Anxiety and Depression scale-MND (HADS-MND) . (22) was used to assess mood disorders in patients. The anxiety and depression subscales range from 0-18 and 0-16, respectively. Lower scores indicate less anxiety or 
depression.

\section{Physical symptoms}

The Symptom Inventory-ALS consists of 6 items, one for each symptom assessed: drooling, choking, head drop, cramps, fasciculations and emotional lability. Each symptom is rated according to three levels: 'I don't have this', 'I have this but it doesn't bother me' and 'I have this and it does bother me' and is treated as a categorical variable in the regression analysis.

\section{Statistical Analysis}

All explanatory variables were standardised before analysis. Significance was assessed using the likelihood ratio criterion with $\mathrm{p}<0.05$ as the cut-off for significance. The analyses were conducted on complete cases only. All analyses were carried out in R version 3.3.1.

Simple linear regression analyses were first conducted to assess the individual associations of age, sex, onset type, bulbar, motor and respiratory functional deficits with social withdrawal. Through forward selection a multiple regression model was then developed and interactions between main effects were tested for significance.

To assess whether physical symptoms, anxiety and depression give additional information for identifying people at risk of social withdrawal, items from the Symptom Inventory-ALS and anxiety and depression scores were added to the multiple regression model for social withdrawal using forward selection. Interaction effects were tested for significance.

The mediating role of stigma was investigated through the development of a joint model for stigma and social withdrawal. In order to estimate the direct effects of 
covariates on social withdrawal after adjustment for stigma, and to distinguish these from indirect effects of covariates on social withdrawal mediated by stigma, the bivariate distribution was factorised according to the mediation hypothesis (see Supplementary Material section S.3 for the conceptual causal pathway). All explanatory variables and interaction terms found to be significant predictors of social withdrawal in the previous analysis were included in the initial bivariate model. Non-significant effects were then removed step-wise by backward elimination.

\section{Results}

\section{Participants}

632 participants were considered in the current analysis. Out of these, 73 individuals were excluded from the analysis due to missing data. Table 1 gives the demographics of the remaining study population $(\mathrm{N}=559)$.

The distributions of social withdrawal and stigma in the study population are shown in Figure 1. Social withdrawal was approximately Normally distributed with a sample mean and median of 24.79 and 25.2 (on a scale from 0-42), respectively, a sample standard deviation of 3.53 and a range of 12.25 to 42 . The remaining study population was located more centrally along the scale thus indicating that most individuals experienced some form of social withdrawal. Section S.4 in the Supplementary Material shows the associations of social withdrawal with age, sex, onset type and stigma as found in the study population.

Stigma appeared to follow a bimodal distribution, with $13 \%$ of the study population not experiencing any stigma and the levels in the remaining population approximating a Normal distribution. The sample mean and median level of stigma 
were 8.85 and 8.36 , respectively, on a scale from 0 to 32 . The sample standard deviation was 5.42. The highest level of stigma was 26.32 .

Table 2 gives the frequencies of the occurrence of those symptoms covered by the Symptom Inventory-ALS and the frequency at which the symptoms caused distress to those affected. Fasciculations and muscle cramps were the most common symptoms, but bothered only a quarter and two fifths of the affected population, respectively. Drooling, choking and emotional lability bothered about half of the individuals affected. Head drop was experienced by only a quarter of the study population, but bothered twothirds of those affected.

Table 3 gives summary statistics of the remaining variables used in the analysis.

\section{Are functional deficits and demographic characteristics predictors of social withdrawal?}

All three domains of ALSFRS-R (bulbar, motor and respiratory) as well as sex and onset-type were significantly associated with social withdrawal in a univariable regression analysis. The multiple regression model showed that social withdrawal increases with worse motor and bulbar function (-0.31 (95\% CI: $-0.39,-0.23) ;-0.31$ (95\% CI: -0.39,-0.24), respectively). Motor and bulbar function also had a small but significant interaction effect on social withdrawal (-0.08 (95\% CI: -0.16, -0.01)), indicating that the negative effect of functional ability in each of the domains on social withdrawal is further enhanced by the functional ability in the second domain. Age had a weak negative association $(-0.09$ (95\% CI: $-0.16,-0.01))$ with social withdrawal after adjusting for the functional deficits. Parameter estimates are given in the Supplementary Material, section S.5 Table 1. Age, motor and bulbar function together explained 19\% of the total variation in social withdrawal in the study population as measured by 
adjusted $\mathrm{R}^{2}$.

\section{Do anxiety and depression scores or the Symptom Inventory-ALS capture additional risk factors for social withdrawal?}

Including anxiety, depression and physical symptoms, as recorded by the Symptom Inventory-ALS, as explanatory variables in the previously developed model for social withdrawal showed that individually, anxiety, depression, emotional lability, fasciculation, muscle cramps and choking are significantly associated with social withdrawal after adjustment for demographics and functional deficits. In the forward selection process, however, none of the symptoms remained significant after adjustment for depression and anxiety. Increased social withdrawal was associated with worse depressive symptoms $(0.26$ (95\% CI: $0.17,0.34))$ and more anxiety $(0.19$ (95\% CI: $0.11,0.27)$ ). Figure 2 shows that social withdrawal increased with anxiety and depression whether or not the patient scores fall above the cut-off levels suggested for possible or probable cases (22). Depression also had a small but significant interaction effect with the motor domain of the ALSFRS-R; the rate at which social withdrawal increases with depression, increases with better functional ability (0.09 (95\% CI: 0.02 , 0.16)). Parameter estimates for the full model are given in the Supplementary Material section S.4 Table 2. Inclusion of depression and anxiety as predictors for social withdrawal increased the adjusted $\mathrm{R}^{2}$ value of the model to $35 \%$.

Could stigma be a mediator for the effects of functional deficits, depression and anxiety on social withdrawal? 
After removing non-significant effects from the joint model for stigma and social withdrawal, direct effects of stigma, bulbar function, motor function, depression and anxiety on social withdrawal remained, as well as effects of motor function, bulbar function, depression, anxiety, and age on stigma. The previously found interaction effect between motor and bulbar function only had a significant effect on stigma. This suggests that the effects of motor and bulbar function on stigma are more than additive whereas this is not the case for their effects on social withdrawal after stigma is adjusted for. The interaction between depression and motor function was only found to have a significant direct effect on social withdrawal such that the rate at which social withdrawal increases with depression, increases with better functional ability, whereas motor function and depression have independent effects on stigma.

Stigma showed the largest effect on social withdrawal (0.34 (95\%CI: 0.27 , 0.42)). There were additional direct effects of comparable sizes for: depression $(0.2$ (95\% CI: $0.11,0.28))$, motor function $(-0.16(-0.23,-0.1))$ and bulbar function $(-0.17$ ($0.24,-0.11))$. The interaction between depression and motor function showed the smallest direct effect (0.09 (95\%CI: 0.03, 0.16)).

Anxiety, bulbar function and age were the three strongest predictors for stigma (0.28 (95\% CI: 0.2, 0.36), -0.24 (95\%CI: -0.31, -0.17) and -0.24 (95\%CI: -0.31, -0.18), respectively). The other predictors, depression (0.18 (95\%CI: $0.1,0.27))$ and motor function (-0.14 (95\%CI: $-0.21,-0.07))$, had marginally smaller effects. The parameter estimates for this model are given in Table 4. The resulting conceptual pathway is shown in Figure 3. Table 5 gives the estimated indirect effects of functional deficits, age, anxiety and depression on social withdrawal through stigma. Stigma mediated $11 \%-41 \%$ of the effect of motor function on social withdrawal and $20 \%$ to $50 \%$ of the effect of bulbar function on social withdrawal. Between $14 \%$ and $41 \%$ of the effect of 
depression on social withdrawal was mediated by stigma and for anxiety $28 \%$ to $90 \%$ of the effect was indirect via stigma.

This final model for social withdrawal had an adjusted $\mathrm{R}^{2}$ value of $42 \%$. 


\section{Discussion}

This is the largest study of social withdrawal in ALS or any neurological condition, as previous literature concerns mental health $(23,24)$ or $\operatorname{HIV}(25,26)$. Social withdrawal is widely experienced, to varying degrees, among this large cohort of people with ALS recruited across the UK when attending for routine clinic follow-up. Using a well validated outcome measure (19), social withdrawal appears to follow a Normal distribution with no participant reporting a floor effect and only one reporting a ceiling effect.

Social withdrawal increases with worse motor or bulbar function, and the synergistic relation between the two impairments places the patient particularly at risk when they experience both. Anxiety and depression scores both significantly increase the risk of social withdrawal, whether or not the patient scores fall above the cut-off levels suggested for possible or probable cases (22). Thus, at levels which would not lead to a diagnosis of either depression or anxiety, feelings of low mood increase the risk of social withdrawal. This relationship may be circular, in that social withdrawal has been reported as part of the experience of depression among people with neurological conditions (27) and incurable illnesses (28) whilst in longitudinal studies of young people, social withdrawal increases the risk of later depression (29). Specific ALS-related symptoms are not associated with more social withdrawal once anxiety and depression are taken into account.

Depression and physical disability interact on social withdrawal in a complex manner; increasing depression is associated with greater social withdrawal but the rate increases with less physical disability. Clinical experience shows that patients who are 
depressed and tending to withdraw socially may have to maintain some social contacts if their physical disability necessitates them relying on others. In routine clinical care, the patient who is particularly vulnerable to severe social withdrawal has bulbar impairment and depression, but sufficiently preserved motor function that they can manage without day-to-day assistance.

Earlier work has shown that stigma is more prevalent in ALS than in multiple sclerosis or Parkinson's disease (13). Patient-perceived stigma is the strongest single predictor for social withdrawal amongst the factors tested here and may partially mediate the effects of functional impairments, anxiety and depression. The joint model shows that increased anxiety, impaired bulbar function, and age are the strongest predictors for greater feelings of stigma. The impact of anxiety on stigma has been identified in large, multinational studies (30). The adverse impact of poor bulbar function on stigma may be related to the stigmatising effects of altered or lost speech, as has been demonstrated in speech disorders like stuttering, or speech loss through laryngectomy (31). Clinical services should monitor younger patients with high levels of anxiety and depression together with loss of speech or swallowing as they appear at particular risk of experiencing stigma and social withdrawal.

Services in the clinical, patient support and voluntary sectors should foster social interaction as a means to ameliorate stigma, depression and social withdrawal. There are no published studies on how to reduce social withdrawal in MND; a recent review on reducing social isolation in older people recommended social activity and/or support within a group format (32). Large, multinational studies of people with depression showed that stigma can co-exist with depression but may be lessened by more social 
contact (33). In a longitudinal intervention study, social identification, or the sense of being part of a group, predicted recovery from depression (34).

In this study we have identified predictors for social withdrawal and have built a case for a causal pathway from symptoms and disabilities to social withdrawal in which self-perceived stigma has a mediating role. However, there are several limitations to consider. Due to the cross-sectional nature of this study it is not possible to establish any causal direction, we rather assess whether an a priori hypothesised causal pathway is in agreement with the data. A second limitation of this study is the omission of potentially confounding variables. Omitted variable bias is not a concern for the identification of predictors for social withdrawal; it may, however, have large effects on the results regarding the causal pathway. The omission of confounding variables may produce spurious causal relationships. In particular, when carrying out mediation analyses, the omission of variables that affect the exposure and the outcomes, the exposure and the mediator, or the mediator and the outcome can give rise to severe biases in the estimated direct and indirect effects (e.g. (35-37)). This may be one reason why previously published work, which included different variables than in this study, did not find a relationship between depression and social withdrawal (3), unlike the results shown here.

Stigma, depression, anxiety, age, bulbar and motor function explain $42 \%$ of the observed variation in social withdrawal. The role of other factors remains uncertain, although research in adults with disabilities suggests that self-esteem is associated with both stigma and social avoidance (38). Stigma has an important role in social withdrawal, and both of these factors have relevance to quality of life. Clinicians should also be mindful about relationships between stigma, anxiety and depression, which may have effects on quality of life beyond those of physical disability alone. 
Future longitudinal analysis will further examine the causal relationships between stigma, anxiety and depression on social withdrawal.

Word Count: 3094

\section{Acknowledgements}

We thank the participants and their families for their invaluable contributions; the research and clinical staff for recruitment, and the TONiC team.

\section{Funding details}

This work was supported by the MS Society, the MNDA, the NIHR CLRN, the Neurological Disability Fund 4530, and unrestricted grants from Biogen, Genzyme, Merck, Novartis, Roche and Teva.

\section{Disclosure of interest}

The authors report no conflicts of interest.

\section{References}

1. Green C, Kiebert G, Murphy C, Mitchell JD, O'Brien M, Burrell A, et al. Patients' health-related quality-of-life and health state values for motor neurone disease/amyotrophic lateral sclerosis. Qual Life Res. 2003;12(5):565-74.

2. van Groenestijn AC, Kruitwagen-van Reenen ET, Visser-Meily JM, van den Berg LH, Schroder CD. Associations between psychological factors and health-related quality of life and global quality of life in patients with ALS: a systematic review. Health Qual Life Outcomes. 2016;14(1):107.

3. Gibbons C, Thornton E, Ealing J, Shaw P, Talbot K, Tennant A, et al. The impact of fatigue and psychosocial variables on quality of life for patients with motor neuron disease. Amyotroph Lateral Scler Frontotemporal Degener. 2013;14(7-8):53745 .

4. Goldstein LH, Atkins L, Leigh PN. Correlates of Quality of Life in people with motor neuron disease (MND). Amyotroph Lateral Scler Other Motor Neuron Disord. 2002;3(3):123-9. 
5. Chio A, Gauthier A, Montuschi A, Calvo A, Di Vito N, Ghiglione P, et al. A cross sectional study on determinants of quality of life in ALS. J Neurol Neurosurg Psychiatry. 2004;75(11):1597-601.

6. Ganzini L, Johnston WS, Hoffman WF. Correlates of suffering in amyotrophic lateral sclerosis. Neurology. 1999;52(7):1434-40.

7. de Boer AG, Wijker W, Speelman JD, de Haes JC. Quality of life in patients with Parkinson's disease: development of a questionnaire. J Neurol Neurosurg Psychiatry. 1996;61(1):70-4.

8. Stenager E, Stenager EN, Knudsen L, Jensen K. Multiple sclerosis: the impact on family and social life. Acta Psychiatr Belg. 1994;94(3):165-74.

9. Coplan RJ, Rubin KH. Social Withdrawal: Definitions and Perspectives. In: LoCoco A, Rubin KH, Zappulla C, editors. L'isolamento sociale durante l'infanzia (Social withdrawal in childhood). Milan, Italy: Unicopli; 2008.

10. McDonald E. Psychological Aspects of ALS Patients and Their Primary Caregivers. The ALS Patient Profile Project1992.

11. Hogg KE, Goldstein LH, Leigh PN. The psychological impact of motor neurone disease. Psychol Med. 1994;24(3):625-32.

12. Crocker J, Major, B., \& Steele, C. M. . Social stigma. In: Gilbert D, Fiske, ST, Lindzey, G, editor. The handbook ofsocial psychology. 2. New York: Oxford University Press; 1998. p. 504-53.

13. Molina Y, Choi SW, Cella D, Rao D. The stigma scale for chronic illnesses 8item version (SSCI-8): development, validation and use across neurological conditions. Int J Behav Med. 2013;20(3):450-60.

14. Gray AJ. Stigma in psychiatry. J R Soc Med. 2002;95(2):72-6.

15. Rasch G. Probabilistic models for some intelligence and achievement tests. Copenhagen: Danish Institute for Educational Research. 1960.

16. da Rocha NS, Chachamovich E, de Almeida Fleck MP, Tennant A. An introduction to Rasch analysis for Psychiatric practice and research. J Psychiatr Res. 2013;47(2):141-8.

17. Marais I, Andrich D. Formalizing dimension and response violations of local independence in the unidimensional Rasch model. J Appl Meas. 2008;9(3):200-15. 18. Hagquist C, Andrich D. Recent advances in analysis of differential item functioning in health research using the Rasch model. Health Qual Life Outcomes. 2017;15(1):181.

19. Gibbons CJ, Thornton EW, Ealing J, Shaw PJ, Talbot K, Tennant A, et al. Assessing social isolation in motor neurone disease: a Rasch analysis of the MND Social Withdrawal Scale. J Neurol Sci. 2013;334(1-2):112-8.

20. Cedarbaum JM, Stambler N, Malta E, Fuller C, Hilt D, Thurmond B, et al. The ALSFRS-R: a revised ALS functional rating scale that incorporates assessments of respiratory function. BDNF ALS Study Group (Phase III). J Neurol Sci. 1999;169(12):13-21.

21. Franchignoni F, Mora G, Giordano A, Volanti P, Chio A. Evidence of multidimensionality in the ALSFRS- R Scale: a critical appraisal on its measurement properties using Rasch analysis. J Neurol Neurosur Ps. 2013;84(12):1340-5.

22. Gibbons CJ, Mills RJ, Thornton EW, Ealing J, Mitchell JD, Shaw PJ, et al. Rasch analysis of the hospital anxiety and depression scale (HADS) for use in motor neurone disease. Health Qual Life Outcomes. 2011;9:82.

23. Schennach R, Riedel M, Obermeier M, Spellmann I, Musil R, Jager M, et al. What are residual symptoms in schizophrenia spectrum disorder? Clinical description 
and 1-year persistence within a naturalistic trial. Eur Arch Psy Clin N. 2015;265(2):10716.

24. Maki P, Koskela S, Murray GK, Nordstrom T, Miettunen J, Jaaskelainen E, et al. Difficulty in making contact with others and social withdrawal as early signs of psychosis in adolescents - the Northern Finland Birth Cohort 1986. Eur Psychiat. 2014;29(6):345-51.

25. Stutterheim SE, Pryor JB, Bos AER, Hoogendijk R, Muris P, Schaalma HP. HIV-related stigma and psychological distress: the harmful effects of specific stigma manifestations in various social settings. Aids. 2009;23(17):2353-7.

26. Hasan MT, Nath SR, Khan NS, Akram O, Gomes TM, Rashid SF. Internalized HIV/AIDS-related Stigma in a Sample of HIV-positive People in Bangladesh. J Health Popul Nutr. 2012;30(1):22-30.

27. Mast BT. Cerebrovascular disease and late-life depression - A latent-variable analysis of depressive symptoms after stroke. Am J Geriat Psychiat. 2004;12(3):315-22. 28. Brenne E, Loge JH, Kaasa S, Heitzer E, Knudsen AK, Wasteson E, et al. Depressed patients with incurable cancer: which depressive symptoms do they experience? Palliative \& Supportive Care. 2013;11(6):491-501.

29. Rubin KH, Coplan RJ, Bowker JC. Social Withdrawal in Childhood. Annu Rev Psychol. 2009;60:141-71.

30. Alonso J, Buron A, Bruffaerts R, He Y, Posada-Villa J, Lepine JP, et al. Association of perceived stigma and mood and anxiety disorders: results from the World Mental Health Surveys. Acta Psychiat Scand. 2008;118(4):305-14.

31. Boyle MP. Identifying correlates of self-stigma in adults who stutter: Further establishing the construct validity of the Self-Stigma of Stuttering Scale (4S). J Fluency Disord. 2015;43:17-27.

32. Dickens AP, Richards SH, Greaves CJ, Campbell JL. Interventions targeting social isolation in older people: a systematic review. BMC Public Health. 2011;11:647. 33. Brohan E, Gauci D, Sartorius N, Thornicroft G, Grp G-ES. Self-stigma, empowerment and perceived discrimination among people with bipolar disorder or depression in 13 European countries: The GAMIAN-Europe study. J Affect Disorders. 2011;129(1-3):56-63.

34. Cruwys T, Haslam SA, Dingle GA, Jetten J, Hornsey MJ, Chong EMD, et al. Feeling connected again: Interventions that increase social identification reduce depression symptoms in community and clinical settings. J Affect Disorders. 2014;159:139-46.

35. Cole SR, Hernan MA. Fallibility in estimating direct effects. Int J Epidemiol. 2002;31(1):163-5.

36. VanderWeele TJ. Mediation Analysis: A Practitioner's Guide. Annu Rev Public Health. 2016;37:17-32.

37. Bullock JG, Green DP, Ha SE. Yes, but what's the mechanism? (don't expect an easy answer). J Pers Soc Psychol. 2010;98(4):550-8.

38. Zhang L, Li W, Liu B, Xie W. Self-esteem as mediator and moderator of the relationship between stigma perception and social alienation of Chinese adults with disability. Disabil Health J. 2014;7(1):119-23.

39. Baron RM, Kenny DA. The moderator-mediator variable distinction in social psychological research: conceptual, strategic, and statistical considerations. J Pers Soc Psychol. 1986;51(6):1173-82.

40. Canty A, Ripley B. boot: Bootstrap R (S-Plus) Functions. . 2017. 
41. Davison AC, Hinkley DV. Bootstrap methods and their application. Cambridge ; New York, NY, USA: Cambridge University Press; 1997. x, 582 p. p. 


\section{Tables}

Table 1: Demographics of the study Population

\begin{tabular}{|l|r|}
\hline \multicolumn{1}{|c|}{ Variable } & \multicolumn{2}{|c|}{ Value/Frequency } \\
\hline $\mathrm{N}$ & $54.72(10.66)$ \\
\hline Age in years (mean (sd)) & $343(61.4)$ \\
\hline Sex = male (\%) & \\
\hline Type of onset (\%) & $397(71.0)$ \\
\hline Motor & $152(27.2)$ \\
\hline Bulbar & $10(1.8)$ \\
\hline Respiratory & $11.79(4.83,30.58)$ \\
\hline $\begin{array}{l}\text { Time since diagnosis in months (median } \\
\text { (IQR)) }\end{array}$ & $77(13.8)$ \\
\hline Employment (\%) & $258(46.2)$ \\
\hline working/student & $128(22.9)$ \\
\hline retired & $6(1.1)$ \\
\hline unable to work due to illness & $9(1.6)$ \\
\hline unemployed & $81(14.5)$ \\
\hline other & $431(77.1)$ \\
\hline NA & $40(7.2)$ \\
\hline Marital status (\%) & $29(5.2$ \\
\hline married & $47(8.4)$ \\
\hline divorced & $12(2.1)$ \\
\hline single & \\
\hline widowed & \\
\hline NA & \\
\hline
\end{tabular}


Table 2: Frequency of observed symptoms as listed in the Symptom Inventory-ALS where 0 indicates 'I don't have it', 1: 'I have it but it doesn't bother me' and 2: 'I have it and it does bother me'.

\begin{tabular}{|c|c|}
\hline Symptom & Frequency \\
\hline \multicolumn{2}{|l|}{ Fasciculations (\%) } \\
\hline 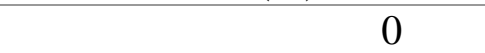 & $106(19.0)$ \\
\hline 1 & $339(60.6)$ \\
\hline 2 & $114(20.4)$ \\
\hline \multicolumn{2}{|l|}{ Cramps (\%) } \\
\hline 1 & $141(25.2)$ \\
\hline 1 & $241(43.1)$ \\
\hline 2 & $177(31.7)$ \\
\hline \multicolumn{2}{|l|}{ Head drop (\%) } \\
\hline 0 & $418(74.8)$ \\
\hline 1 & $47(8.4)$ \\
\hline 2 & $94(16.8)$ \\
\hline \multicolumn{2}{|l|}{ Drooling (\%) } \\
\hline 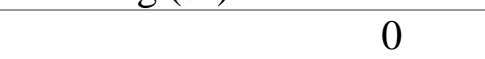 & $345(61.7)$ \\
\hline 1 & $111(19.9)$ \\
\hline 2 & $103(18.4)$ \\
\hline \multicolumn{2}{|l|}{ Choking (\%) } \\
\hline 0 & $324(58.0)$ \\
\hline 1 & $116(20.8)$ \\
\hline 2 & $119(21.3)$ \\
\hline \multicolumn{2}{|l|}{ Emotional Lability (\%) } \\
\hline 0 & $347(62.1)$ \\
\hline 1 & $108(19.3)$ \\
\hline 2 & $104(18.6)$ \\
\hline
\end{tabular}


Table 3: Summary statistics of the distributions of depression, anxiety and functional ability in the study population.

\begin{tabular}{|l|r|r|r|}
\hline \multicolumn{1}{|c|}{ Variable } & Range of the scale & Observed Median & Observed 25\%ile, 75\%ile \\
\hline Depression & $0-16$ & 5.73 & $2.32,8.26$ \\
\hline Anxiety & $0-18$ & 5.41 & $3.25,7.85$ \\
\hline ALSFRS-R bulbar & $0-12$ & 8.16 & $5.89,12$ \\
\hline ALSFRS-R motor & $0-24$ & 13.19 & $9.85,15.78$ \\
\hline ALSFRS-R respiratory & $0-12$ & 11.09 & $9.55,12$ \\
\hline
\end{tabular}


Table 4: Tables of the standardised parameters estimates for the model of the joint distribution of social withdrawal and stigma conditional on demographics, functional deficits, anxiety and depression (including p-values and 95\% confidence intervals)

\begin{tabular}{|c|c|c|c|c|}
\hline & Estimate & P-value & $2.5 \%$ & $97.5 \%$ \\
\hline \multicolumn{5}{|c|}{ stigma } \\
\hline Intercept & 0.00 & $9.66 \mathrm{E}-01$ & -0.07 & 0.07 \\
\hline ALSFRS-R motor & -0.14 & $1.11 \mathrm{E}-04$ & -0.21 & -0.07 \\
\hline ALSFRS-R bulbar & -0.24 & $1.83 \mathrm{E}-11$ & -0.31 & -0.17 \\
\hline age & -0.24 & $5.81 \mathrm{E}-12$ & -0.31 & -0.18 \\
\hline depression & 0.18 & $3.90 \mathrm{E}-05$ & 0.10 & 0.27 \\
\hline anxiety & 0.28 & $1.28 \mathrm{E}-10$ & 0.20 & 0.36 \\
\hline $\begin{array}{l}\text { ALSFRS-R motor: } \\
\text { ALSFRS-R bulbar }\end{array}$ & -0.09 & $9.27 \mathrm{E}-03$ & -0.16 & -0.02 \\
\hline \multicolumn{5}{|c|}{ social withdrawal } \\
\hline Intercept & 0.03 & $4.40 \mathrm{E}-01$ & -0.04 & 0.09 \\
\hline stigma & 0.34 & $5.25 \mathrm{E}-18$ & 0.27 & 0.42 \\
\hline ALSFRS-R motor & -0.16 & $2.38 \mathrm{E}-06$ & -0.23 & -0.10 \\
\hline ALSFRS-R bulbar & -0.17 & $3.13 \mathrm{E}-07$ & -0.24 & -0.11 \\
\hline depression & 0.20 & $3.95 \mathrm{E}-06$ & 0.11 & 0.28 \\
\hline anxiety & 0.10 & $2.12 \mathrm{E}-02$ & 0.01 & 0.18 \\
\hline $\begin{array}{l}\text { depression: } \\
\text { ALSFRS-R motor }\end{array}$ & 0.09 & $3.55 \mathrm{E}-03$ & 0.03 & 0.16 \\
\hline
\end{tabular}


Table 5: Table of the estimated indirect effects of function deficits, age, depression and anxiety on social withdrawal through sigma. Effects are calculated as a product of the effect of the covariate on stigma and the effect of stigma on social withdrawal (39). 95\% Confidence intervals are based on non-parametric bootstrap and are calculated using the R package "boot" and the adjusted percentile method therein $(40,41)$.

\begin{tabular}{|c|c|c|c|}
\hline & Estimate & $2.5 \%$ & $97.5 \%$ \\
\hline ALSFRS-R motor & -0.05 & -0.08 & -0.02 \\
\hline ALSFRS-R bulbar & -0.08 & -0.12 & -0.05 \\
\hline age & -0.08 & -0.13 & -0.05 \\
\hline depression & 0.06 & 0.03 & 0.11 \\
\hline anxiety & 0.1 & 0.05 & 0.15 \\
\hline $\begin{array}{l}\text { ALSFRS-R motor: } \\
\text { ALSFRS-R bulbar }{ }^{1}\end{array}$ & -0.03 & -0.06 & -0.01 \\
\hline
\end{tabular}

